\title{
Clarificaciones nomenclaturales y CiRCunscripción taXonómica de Schinus areira (AnACARdiaceae) en Argentina
}

\author{
MARIA A. ZAPATER ${ }^{1 *}$, VÍCTOR H. AQUINO ${ }^{1}$, CAROLINA B. FLORES ${ }^{1}$ y \\ EVANGELINA C. LOZANO ${ }^{1}$
}

\begin{abstract}
Summary: Nomenclatural clarifications and taxonomic circumscription of Schinus areira (Anacardiaceae) in Argentina. Schinus areira, an originally South American species, is naturalized in the northwest and west of Argentina, and has a great morphological affinity with S. molle, with which it shares part of its distribution range. Throughout their taxonomic history, both species have been treated as independent species, as a single species with two varieties or synonymized under the same taxon. The objective of this work was to review the taxonomy of $S$. areira, to redefine the diagnostic characters of the morphology that clearly differentiate it from S. molle in Argentina, and to perform nomenclatural clarifications. As a result, the specimens deposited in the LINN herbarium are discarded as possible types and the name of $S$. areira is lectotypified by means of an illustration. On the other hand, the following vegetative characters of $S$. areira were identified with taxonomic value: leaf dimorphism in the same branch, presence of wings in the rachis, and number of pairs and margin of leaflets. Additionally, a dichotomous key is presented to separate $S$. areira from S. molle.
\end{abstract}

Key words: Anacardiaceae, lectotypification, nomenclature, Schinus molle, taxonomy.

\begin{abstract}
Resumen: Schinus areira, especie originalmente sudamericana, se encuentra naturalizada en el noroeste y oeste de Argentina, y presenta una gran afinidad morfológica con S. molle con quien comparte parte de su rango de distribución. A lo largo de su historia taxonómica, ambas especies han sido tratadas como especies independientes, como una misma especie con dos variedades o sinonimizadas bajo el mismo taxón. El objetivo de este trabajo fue revisar la taxonomía de S. areira, redefinir los caracteres diagnósticos de la morfología que permitan diferenciarla claramente de S. molle en Argentina, y realizar clarificaciones nomenclaturales. Como resultado se descartan como posibles tipos los especímenes depositados en el herbario LINN y se lectotipifica el nombre de S. areira por medio de una ilustración. Por otro lado, se identificaron los siguientes caracteres vegetativos de S. areira con valor taxonómico: dimorfismo foliar en la misma rama, presencia de alas en el raquis y número de pares y margen de folíolos. Adicionalmente se presenta una clave dicotómica para separar S. areira de S. molle.
\end{abstract}

Palabras clave: Anacardiaceae, lectotipificación, nomenclatura, Schinus molle, taxonomía.

\section{INTRODUCCIÓN}

La especie Schinus areira L. (Anacardiaceae, Anacardioideae) fue descripta por Linneo en el año 1753 simultáneamente con Schinus molle L., morfológicamente muy afín. A partir de entonces ambos taxones han sido tratados como especies independientes, como dos variedades de una única especie o como la misma especie, generándose conflictos respecto a su correcta identidad taxonómica. En 1825, De Candolle fue el primero en considerar a $S$. areira como una variedad de $S$. molle, concepto posteriormente aceptado por Cabrera (1938) y Barkley (1957). De acuerdo a Martínez-Crovetto (1963), ambos taxones son claramente especies diferentes, basándose principalmente en características florales y

\footnotetext{
${ }^{1}$ Facultad de Ciencias Naturales y Consejo de Investigación, Universidad Nacional de Salta, Av. Bolivia 5150, 4400 Salta, Argentina.

*aliciazapater@yahoo.com.ar
} 
foliares. Sin embargo, Legname (1982) mantuvo a $S$. areira como variedad de $S$. molle. Por su lado, Muñoz (1990, 2000) en el marco de la Flora del Paraguay y para Argentina delimitó a $S$. areira y $S$. molle en base a caracteres foliares poco claros y con solapamientos como número de folíolos, la disposición, la forma y el tipo de ápices. Asimismo, $S$. areira fue citada $y$ descripta en floras provinciales de Argentina, con caracteres que a menudo no la delimitan claramente respecto a $S$. molle, como las de San Juan (Múlgura, 2003), Salta en el Valle de Lerma (Juárez de Varela \& Novara, 2007) y Mendoza (Martínez Carretero, 2009).

Otro problema que presenta la especie es sobre su origen. Para Linneo (1753), según consta en el protólogo, la localidad tipo de $S$. areira es en Perú. Más recientemente, según Demaio et al. (2015), $S$. areira sería una especie de origen peruano con naturalización en Argentina, Bolivia y Chile. Esta especie representaba al Inca y por ello se plantaba a la vera de las rutas de comunicación del imperio incaico para prestar abrigo a los chasquis (Demaio et al., 2002). La especie habría llegado también a Norteamérica, según cita del sacerdote jesuita J. De Acosta en 1950 (Demaio et al., 2002, 2015).

Respecto a la morfología de $S$. areira, hasta el momento sus descripciones, a veces como variedad de $S$. molle, resultan generalmente incompletas sin la mención de las flores perfectas (Cabrera, 1938; Barkley, 1957; Legname, 1982; Muñoz, 2000), salvo la de Juárez de Varela \& Novara (2007). Incluso, por ejemplo para Demaio et al. $(2002,2015)$ la especie es dioica con flores imperfectas dispuestas en pies separados.

A pesar de los variados antecedentes que existen sobre $S$. areira, no hay acuerdo sobre su identidad taxonómica y las diferencias morfológicas entre $S$. areira y S. molle no son claras, por lo que se considera necesario revisar nuevamente los caracteres utilizados para delimitarlas y de ser posible, ampliarlos. Por lo expuesto, esta investigación tiene como objetivo revisar la taxonomía de $S$. areira, redefinir los caracteres diagnósticos de la morfología que permitan diferenciarla claramente de S. molle en Argentina, y realizar clarificaciones nomenclaturales.

\section{Materiales y Métodos}

Se consultaron las imágenes digitales de los ejemplares de Linneo depositados en el Herbario LINN que son considerados ejemplares originales de $S$. areira y $S$. molle. Por otra parte, se estudiaron las colecciones de Argentina de ambas especies, existentes en los herbarios CTES, LIL y MCNS. Además se efectuaron nuevas colecciones de $S$. areira en zonas serranas de Jujuy, Mendoza y Salta, tanto en ambientes naturales como cultivados.

En cada individuo/espécimen se analizaron caracteristicas cuantitativas: diámetro altura de pecho, altura total y de fuste, largo y ancho de folíolos, y número de pares o sub-pares y total de folíolos. Por otro lado, se analizaron las características cualitativas como disposición, márgenes y ápices de los folíolos. Para determinar las características florales (cualitativas y cuantitativas) se colectaron por separado flores pistiladas, estaminadas y perfectas en antesis provenientes de individuos diferentes, tomadas al azar. Algunas fueron diseccionadas bajo un microscopio estereoscópico y cada una de sus piezas fueron medidas en $\mathrm{mm}$ con un calibre digital hasta saturación de datos; otras fueron fijadas en FAA (formol, alcohol, ácido acético) para su posterior análisis más detallado en el Microscopio Electrónico de Barrido (MEB; JEOL JSM-6480 LV). Las flores se deshidrataron en una serie creciente de alcoholes y fueron secadas por punto crítico (D’Ambroggio de Argüeso, 1986) en DCP-1 Critical Point-Denton Vacuum. Las muestras fueron montadas en tacos de aluminio, recubiertas con oro con un equipo Denton Vacuum Desk IV.

\section{Resultados}

\section{Tratamiento taxonómico}

Schinus areira L. Sp. Pl. 1: 389. 1753. ESchinus molle L. var. areira (L.) DC. Prodr. 2: 74. 1825. Tipo: Feuillée, F., J. Obs. 3: pl. 30 (Lectotipo, aquí designado). Fig. 1. A-K.

Árbol de 4-8 m de alto y tronco de $15-40 \mathrm{~cm}$ de diám., a veces arbusto achaparrado, corteza rugosa, fisurada, exfoliante, color marrón oscuro, ramas péndulas, con copa irregular, perennifolio, dioico o polígamo, aromático, resinoso. Hojas generalmente imparipinnadas, con pecíolos de 3-7 cm long., no alados, raquis de 10-14 cm long., subalado, (8-)10$15(-22)$ pares y subpares de folíolos, ovados de 2-5,3 x $0,3-0,7 \mathrm{~cm}$, algo asimétricos, opuestos, subopuestos o alternos en las hojas adultas de posición distal en las ramas péndulas, margen generalmente 

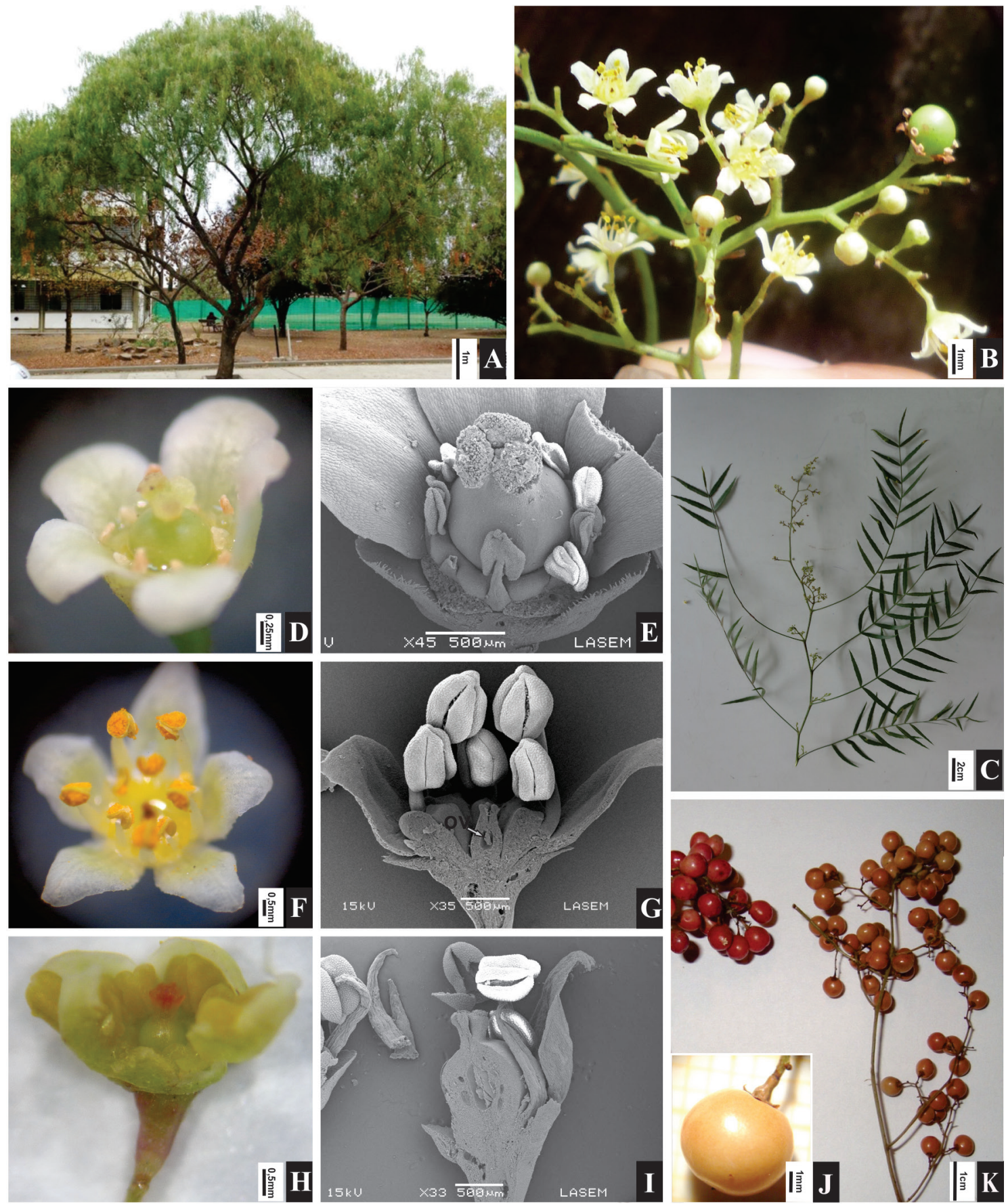

Fig. 1. A-K. Schinus areira. A: Hábito. B: Panícula. C: Rama florífera. D: Flor pistilada, vista superior. E: Flor pistilada, vista superior (MEB). F: Flor estaminada, vista superior. G: Flor estaminada, transcorte longitudinal (MEB), pistilodio con un óvulo incipiente (ov). H: Flor perfecta. I: Flor perfecta, transcorte longitudinal (MEB). J: Fruto. K: Panícula con drupas. Escalas= A: $1 \mathrm{~m} ; \mathrm{B}, \mathrm{J}: 1 \mathrm{~mm} ; \mathrm{C}: 2 \mathrm{~cm}$; D: 0,25 mm; E, G, I: $500 \mu \mathrm{m} ; \mathrm{F}, \mathrm{H}: 0,5 \mathrm{~mm} ; \mathrm{K}: 1 \mathrm{~cm}$. Fotos: A-D, F, H, J-K por V. Aquino; E, G, I por C. A. Gómez. 
entero, raro con algunos dientes; hojas proximales de las ramas péndulas, con folíolos de 3,5-6 x 0,6-0,8 cm, y margen parcialmente aserrado o dentado. Panículas péndulas de 12-60 cm long., con una panícula terminal y 2-15 panículas basales, axilares, muy ramificadas. Flores pentámeras, las pistiladas con sépalos triangulares, de 0,57-0,6 x 0,4-0,42 mm, con ápice romo y densamente ciliado; pétalos oblongos, de 2-2,2 x 0,8-1,2 mm, deflexos en la mitad apical, glabros; disco nectarífero interestaminal con lóbulos de 0,24-0,26 mm long.; estaminodios 10 , filamentos de $0,4-0,43 \mathrm{~mm}$ long. y anteras dorsifijas de 0,38-0,4 mm long.; gineceo de ca. $1,5 \mathrm{~mm}$ long., ovario de 0,86-0,93 mm diám., estilo único, corto de 0,29-0,3 x 0,26$0,27 \mathrm{~mm}$, con tres cabezas estigmáticas grandes de 0,21-0,34 x 0,31-0,39 mm. Flores estaminadas con sépalos triangulares, de 0,8-0,83 x 0,4-0,5 mm, ápice agudo y margen con cilios esparcidos; pétalos obovados, de 1,5-1,8 x 0,88-0,9 mm, deflexos, glabros; lóbulos del disco nectarífero de 0,8-0,9 $\mathrm{mm}$ long., estambres en dos ciclos pentámeros, ciclo externo largo con filamentos de 1,7-1,8 mm long. y anteras de 0,7-0,72 mm long., ciclo interno corto con filamentos de 1,3-1,35 mm long. y anteras de 0,4-0,44 mm long.; pistilodio ovado de 0,65-0,7 mm long., ovario de $0,49-0,5 \times 0,42-0,43 \mathrm{~mm}$ con 3 estilos cilíndricos, de 0,2-0,22 x 0,072-0,09 mm, huecos. Flores perfectas raras, androceo y gineceo de longitud similar, gineceo de 1,31-1,33 x 0,8$0,83 \mathrm{~mm}$, con 3 estilos cilíndricos, de 0,3-0,32 x 0,17-0,18 mm, y cabezas estigmáticas del mismo diámetro que el estilo. Drupa esférica, de 4-5 mm diám., amarillenta, lilacina o rojiza. Semilla de 1,22 x $2,78 \mathrm{~mm}$, amarillenta.

Nombres comunes: "Aguaribay”, "árbol de pimienta", "bálsamo", “curunday”, "gualeguay”, "molle", "molle blanco", "molle castilla", "pimentero", "terebinto" (Tortorelli, 2009).

Distribución y hábitat: Habita originalmente en Perú, Bolivia y norte de Chile. En Argentina se considera naturalizada y actualmente se distribuye en el Chaco Serrano, la Prepuna y el Monte, en las provincias de Catamarca, Jujuy, La Rioja, Mendoza, Salta, San Juan, San Luis y Tucumán, entre los 1800-3000 m s.m. (Muñoz, 2000; observaciones propias). Especie pionera aún en suelos pedregosos y salinos, tolerante al frío. Extensamente cultivado en muchas provincias argentinas por su rusticidad y rápido crecimiento (Muñoz, 2000). También ha sido citado en cultivo para México, Colombia y Ecuador (Barkley, 1957).

Fenología: En las poblaciones estudiadas silvestres y cultivadas, el inicio de floración se produjo a mediados de agosto y hasta comienzos de setiembre; la formación de flores estaminadas fue previa a las flores pistiladas en 2-3 semanas. El pico de fructificación se observó en noviembre y los frutos se encontraban maduros a mediados de diciembre.

Debido a que $S$. areira no posee un holotipo designado, y siendo los únicos materiales originales existentes conocidos para esta especie las ilustraciones de Margraf (1648) y Feuillée (1725), se intentó realizar la lectotipificación del nombre a partir de los ejemplares LINN 1193.2 y LINN 1193.3 designados de esta manera en los pliegos de herbario. Si bien ambos ejemplares coinciden exactamente con las características morfológicas foliares de un individuo pistilado con frutos inmaduros de $S$. areira, no fue posible utilizarlos como lectotipo por la incerteza respecto a su procedencia. De acuerdo a nuestro análisis no son materiales originales de $S$. areira y por ello no resultan adecuados como tipos nomenclaturales según el Linnean Plant Name Typification Project's. Por esta circunstancia y al no existir en LINN otros materiales originales de Linneo que se correspondan con la especie (M. Spencer, com. pers.), no se pudo realizar la lectotipificación pretendida con un ejemplar de herbario. Por este motivo y al no encontrarse sintipos, siguiendo el Código Internacional de Nomenclatura (Turland et al., 2018), se propone en este trabajo como lectotipo la ilustración de Feuillée (1725, tabula 30) mencionada en el protólogo, que representa adecuadamente la morfología foliar descripta para la especie.

Respecto al ejemplar LINN 1193.1, actual lectotipo de $S$. molle, se considera inadecuado como tipo por tratarse de una muestra proveniente de un ejemplar cultivado en Holanda (M. Spencer, com. pers.). Como resultado del análisis morfológico de este ejemplar, se observó que si bien posee folíolos totalmente aserrados, no correspondería a $S$. molle por el número de pares y subpares de folíolos que fue de aproximadamente 15. Por otro lado, debido 


\section{A. Zapater et al. - Nomenclatura y taxonomía de Schinus areira en Argentina}

a la disposisión opuesta, subopuesta y alterna de los folíolos este espécimen concordaría más con algunos ejemplares raros de $S$. areira encontrados en ambientes naturales de Argentina en Jujuy (Meyer 3849, LIL) y Salta (Venturi 5374, LIL).

Como resultado del análisis foliar de las colecciones de herbario, de las colectas propias realizadas y de los ejemplares de la población cultivada estudiada se demostró la existencia de dimorfismo foliar en $S$. areira entre las hojas adultas proximales y las distales de las ramas péndulas, en tanto que en $S$. molle las hojas proximales y distales de las ramas son semejantes. Las dimensiones de los folíolos en hojas adultas de $S$. molle fueron algo mayores en largo y en ancho $(4-6,5 \times 0.5-1 \mathrm{~cm})$ que las dimensiones de los folíolos de las hojas de las ramas proximales de $S$. areira (3,5-6 x 0,6-0,8 cm), mientras que los folíolos de las hojas distales de esta especie fueron en general más cortas y delgadas (2$5,3 \times 0,3-0,7 \mathrm{~cm})$. Respecto al número de pares de folíolos, las hojas adultas de $S$. molle presentaron de 4 a 7 pares de folíolos siempre opuestos, en tanto que $S$. areira tuvo generalmente 10 a 15 pares o subpares, variable entre 8-22, con disposición subopuesta y alterna en el sector proximal del raquis y generalmente opuesta en la porcion distal y media, a veces indistintamente. En cuanto al margen de los folíolos, en $S$. molle fue siempre completa y regularmente aserrado; mientras que en $S$. areira predominó el margen entero en los folíolos de hojas distales, raro con algunos dientes; en tanto que en la hojas proximales, los folíolos presentaron más frecuentemente un aserrado parcial, hasta un dentado casi total. Solo muy raramente (dos casos observados) el margen resultó aserrado en todos los folíolos. El ápice en $S$. molle fue generalmente acuminado recto o curvado, a veces apiculadocurvado; en $S$. areira generalmente fue agudo o apiculado-curvado, muy raro acuminado. El raquis foliar en $S$. areira se considera subalado por presentar una prolongación membranácea muy delgada, mientras que en $S$. molle el raquis es circular, isodiamétrico.

Material estudiado: ARGENTINA. Prov. Catamarca: Dpto. Valle Viejo, San Isidro, 26-XI1950, Brizuela 1502 (LIL). Prov. Jujuy: Dpto. Gral. Manuel Belgrano, Yala, 16-V-1948, Sotelo 2013 (LIL); Dpto. Ledesma, Sierra de Calilegua, 800 m s.m., 13-X-1922, Venturi 5374 (LIL);
Dpto. Tilcara, Maimará, 2560 m s.m., 10-I-1912, Barkley 17768 (LIL); a $1 \mathrm{~km}$ al W de Ruta 9, 2450-2700 m s.m., 28-XII-1989, Novara \& Bruno 9234 (MCNS); Dpto. Tumbaya, entre Tumbaya y Chañarcito, a orillas del río Grande, 25-IV2001, Slanis \& Bulacio 720 (LIL); Purmamarca, 12-VI-2016, Zapater et al. 5132 (MCNS). Prov. Mendoza: Dpto. Las Heras, Papagayos, 12-IV1942, Ruiz Leal 8014 (LIL); Dpto. Luján de Cuyo, Facultad de Ciencias Agrarias, cultivado en parque, 20-IX-2017, Zapater et al. 5459 (MCNS); Embalse Potrerillos, 23-IX-2017, Zapater \& Benci 5465 (MCNS). Prov. Salta: Dpto. Cafayate, márgenes del río Chuscha, 1600 m s.m., II-1960, Villa Carenzo 1305 (LIL); ruinas de Chuschapata, $2 \mathrm{~km}$ al SE Banda de Arriba, 1800 m s.m., 22-I2007, Tolaba 4176 (MCNS); Dpto. Capital, ciudad de Salta, Cerro San Bernardo, 12-IX-1976, Zapata 67 (MCNS); campus Universidad Nacional de Salta, árbol No 19 , cultivado, 15-III-2016, Zapater et al. 5100 (MCNS); ídem, árbol $\mathrm{N}^{\circ}$ 4, cultivado, 23-III-2016, Zapater et al. 5107 (MCNS); ídem, árbol 26 cultivado, 25-VIII-2016, Zapater et al. 5163 (MCNS); ídem, árbol $\mathrm{N}^{\circ} 12$, cultivado, $25-$ VIII-2016, Zapater et al. 5165 (MCNS); ídem, árbol $\mathrm{N}^{\circ} 24$, cultivado, 25-VIII-2016, Zapater et al. 5166 (MCNS); Dpto. Iruya, camino de San Isidro a Iruya, $22^{\circ} 46^{\prime} 36,2^{\prime}$ S $65^{\circ} 12^{\prime} 47,6^{\prime \prime} \mathrm{W}, 14$-XII2014, Muruaga et al. 1902 (LIL); alrededores de Iruya, 26-II-1997, Tolaba \& Ragno 802 (MCNS); Dpto. Molinos, Seclantás, 7-I-2018, Zapater et al. 5601 (MCNS); Dpto. Rosario de Lerma, Gólgota, 2000 m s.m., 17-I-1941, Meyer 3849 (LIL); Quebrada del Toro, Ruta 51, caserío al lado del puente, $24^{\circ} 45^{\prime} 25,4^{\prime}$ S $65^{\circ} 44^{\prime} 48,5^{\prime \prime} \mathrm{W}, 30$-VI2016, Zapater et al. 5145 (MCNS); Gobernador Solá, 30-VI-2016; Zapater et al. 5147 (MCNS); Dpto. La Viña, Brealito, $2 \mathrm{~km}$ antes del pueblo, 7-V-1987, Novara 6819 (MCNS); Ruta Nac. 68, Km 46,8, Garganta del Diablo, 6-VII-1985, Novara 4612 (MCNS); Dpto. Santa Victoria, Santa Victoria, 2385 m s.m., 1-II-1943, Meyer 4885 (LIL). Prov. San Juan: Dpto. Capital, Concepción, 15-XI-1945, Cuezzo 1108 (LIL); Dpto.Chimbas, Desamparados, 16-XI-1945, Cuezzo 1152 (LIL); Dpto. Pocito, La Rinconada, 21-XI-1945, Cuezzo 1343 (LIL); Dpto. Santa Lucía, Santa Lucía, 28XII-1945, Cuezzo 2212 (LIL); Dpto. 25 de Mayo, Algarrobo Verde, 7-XII-1945, Cuezzo 1777 (LIL). Prov. San Luis: Dpto. Juan Martín de Pueyrredón, Alto Pencoso, 27-III-1947, Terribile 781 (LIL). 
Material estudiado de Schinus molle: ARGENTINA. Prov. Corrientes: Dpto. Salada, Colonia Cabral, 400 m s.m., 15-XII- 1949, Schwarz 9163 (CTES, LIL, MCNS); Dpto. Santo Tomé, vuelta del Ombú, $3 \mathrm{~km} \mathrm{SW}$ de Gobernador Virasoro, 22-XI-1989, Tressens et al. 3785 (CTES, LIL). Prov. Entre Ríos: Dpto. Capital, Paraná, 23-XII1946, Terribile 612 (LIL). Prov. Misiones: Dpto. Apóstoles, sin localidad, 27-I-1926, Clos 1962 (LIL); Dpto. Caingüas, Ruta 105, 240 m s.m., 2-VI-1950, Sehwindt 4486 (LIL); Oro Verde, 350 m s.m., 28-VIII-1950, Schwarz 10730 (LIL); Dpto. Concepción, Concepción de la Sierra, 400 m s.m., 12-XII-1946, Schwarz 3600 (LIL); Dpto. Iguazú, El Dorado, 1600 m s.m., 4-X-1949, Schwindt 2175 (LIL); Dpto. San Ignacio, Gobernador Roca, 300 m s.m., 8-IX-1945, Schwarz 1230 (LIL); Arroyo Yabebiry, 600 m s.m., 20-IX-1945, Schwarz 1278 (LIL); Dpto. Santa Ana, 22-VI-1909, Venturi 95 (LIL). Prov. Santa Fe: Dpto. Las Colonias, 10-X1991, Hilgert 109 (MCNS).

\section{Clave para la diferenciación de Schinus areira y $S$. molle}

1. Hojas adultas dimorfas en la misma rama, con (8-)10-15(-22) pares y subpares de folíolos opuestos, subopuestos y alternos; las hojas distales con folíolos de margen entero, raro con algunos dientes; las hojas proximales a veces con margen aserrado parcial o dentado casi total. Raquis muy estrechamente alado. S. areira

1' Hojas adultas isomorfas, con 4-7 pares de folíolos opuestos, completamente aserrados. Raquis no alado. S. molle

\section{Discusión}

En Argentina, Schinus areira y S. molle pueden delimitarse claramente sobre la base del número de pares o sub-pares de folíolos y su disposicion, además de la forma del raquis alado o no. Aunque Múlgura (2005) menciona que $S$. molle puede tener hojas con hasta ocho pares de folíolos, en todos los ejemplares revisados nunca se contabilizó este número siendo el máximo siete pares. Por otro lado, el margen entero sería un carácter diferencial en $S$. areira y solo válido para los folíolos de hojas distales, aunque en algunos casos poco frecuentes y raros, todos los folíolos pueden presentar margen dentado e inclusive completamente aserrado. El ápice acuminado es el más común en $S$. molle y el agudo o apiculado-curvado en $S$. areira pero no puede considerarse como un carácter que permita una segura identificación. Según Múlgura (2003) el fruto puede tener un tamaño de 5-6 mm, que es algo mayor al medido en este trabajo.

\section{Conclusión}

Las poblaciones argentinas de $S$. areira se pueden delimitar más ajustadamente de las de $S$. molle a través de nuevos caracteres diagnósticos foliares aportados aquí. Esto cobra importancia porque ambas especies se hallan extensamente cultivadas en las mismas áreas de Argentina. Por el contrario en los ambientes naturales de nuestro país las dos especies tienen una distribución alopátrica lo cual simplifica su reconocimiento. De todas formas, futuros estudios del área completa de la distribución de las especies y moleculares podrán aportar mayores elementos sobre la circunscripción taxonómica, variabilidad morfológica y relaciones filogenéticas de estas dos especies.

\section{Agradecimientos}

Queremos manifestar nuestro profundo agradecimiento a los curadores de los herbarios LIL y MCNS, Dra. Nora Muruaga y Dra. Olga G. Martínez, por su gentil atención. Agradecemos especialmente al personal del Herbario LINN, Lynda Brooks e Isabelle Charmaine, por enviarnos imágenes digitales de materiales linneanos y buscar por nosotros otros ejemplares aptos para tipificación. Un especial reconocimiento a Mark Spencer, curador honorario de dicho herbario, que con total gentileza nos remitió datos de las colecciones, nos informó acerca de aspectos vinculados a los origenes de los ejemplares y nos asesoró sobre su aptitud para ser utilizados como tipos. También a Liliana Mallo de la Biblioteca del Instituto de Botánica Darwinion por ayudarnos en la búsqueda de ilustraciones originales.

Finalmente dedicamos esta producción a quien fue nuestra compañera, la Dra. Patricia Hoc, investigadora de la UBA y el CONICET, que nos 


\section{A. Zapater et al. - Nomenclatura y taxonomía de Schinus areira en Argentina}

asesoró en los trabajos iniciales de esta investigación antes de su fallecimiento en Febrero de 2017, como codirectora del proyecto de investigación en el que participamos y comprende a las Anacardiaceae de Argentina, en cuyo marco investigamos la temática de esta publicación.

\section{Bibliografía}

BARKLEY, F. A. 1957. Schinus L. Lilloa 28: 4-109.

CABRERA, A. L. 1938. Revisión de las Anacardiáceas austroamericanas. Revista Mus. La Plata 2: 3-65.

CORKIDI, L., S. CACHO \& A. BURQUEZ. 1991. Dispersión del pirú (Schinus molle L., Anacardiaceae) por aves en Teotihuacán, México. Acta Bot. Mex. 15: $17-22$

D’AMBROGIO DE ARGÜESO, A. 1986. Manual de Técnicas en Histología Vegetal. Hemisferio Sur, Buenos Aires.

DEMAIO, P., U. O. KARLIN \& M. MEDINA. 2002. Arboles Nativos del Centro de Argentina. L.O.L.A., Buenos Aires.

DEMAIO, P., U. O. KARLIN \& M. MEDINA. 2015. Arboles nativos de Argentina: Centro y Cuyo. Ecoval Ediciones, Córdoba.

FEUILLÉE, L. E. 1725. Journal des observationes physiques, mathematiques et botaniques, t. 3 . Mariette, Paris.

JUÁREZ DE VARELA, F. \& L. J. NOVARA. 2007. Anacardiaceae. En: NOVARA, L. J. (ed.), Flora del Valle de Lerma 8: 1-27.

LEGNAME, P. R. 1982. Arboles indígenas del Noroeste Argentino. Opera Lilloana 34: 65-66.

MARGGRAF, G. 1648. Historia rerum naturalium Brasiliae. En: LAET, J. de (ed.), Historia Naturalis Brasiliae: 1-293. Hackium, Leiden; Elzevirium, Amsterdam.

MARTÍNEZ CARRETERO, E. 2009. Anacardiaceae Lindl. Multequina 18: 1-12.
MARTÍNEZ-CROVETTO, R. 1963. Estudio taxonómico-biométrico de Schinus molle y $S$. areira (Anacardiaceae). Bonplandia 1: 225-244.

MÚLGURA, M. E. 2003. Anacardiaceae. En: KIESLING, R. (ed.), Flora de San Juan 2: 86-90. Estudio Sigma, Buenos Aires.

MÚlgURA, M. E. 2005. Anacardiaceae. En: BACIGALUPO, N. M. (ed.), Flora Ilustrada de Entre Ríos 6 (4b): 195-208. Colección Científica del INTA, Buenos Aires.

MUÑOZ, J. D. D. 1990. Anacardiaceae. En: SPICHIGER, R. \& L. RAMELLA (eds.), Flora del Paraguay: 7-84. Conservatoire et Jardin botaniques de la Ville de Genève, Ginebra.

MUÑOZ, J. de D. 2000. Anacardiaceae. En: HUNZIKER, J. \& A. ANTON (eds.), Flora Fanerogámica Argentina 65: 1-28. Programa PROFLORACONICET, Córdoba.

NASIR, Y. J. 1983. Anacardiaceae. En: NASIR, E. \& S. I. ALI (eds.), Flora of Pakistan 152: 1-22. University of Karachi y Pakistan Agricultural Research Council, Islamabad.

TORTORELLI, L. 2009. Maderas y Bosques Argentinos, 2. Orientación Gráfica Editora, Buenos Aires.

TURLAND, N. J., J. H. WIERSEMA, F. R. BARRIE, W. GREUTER, D. L. HAWKSWORTH, P. S. HERENDEEN, S. KNAPP, W.-H. KUSBER, D.-Z. LI, K. MARHOLD, T. W. MAY, J. MCNEILL, A. M. MONRO, J. PRADO, M. J. PRICE \& G. F. SMITH (eds.). 2018. International Code of Nomenclature for algae, fungi, and plants (Shenzhen Code) adopted by the Nineteenth International Botanical Congress Shenzhen, China, July 2017. Regnum Vegetabile 159. Koeltz Botanical Books, Glashütten. Disponible en: https://www.iapt-taxon.org/nomen/ page/main/art_9.html [acceso: 30 agosto 2018 ].

Recibido el 2 de agosto de 2018, aceptado el 3 de septiembre de 2018. Editor: Diego Gutiérrez. 
